九州大学学術情報リポジトリ

Kyushu University Institutional Repository

\title{
Evaluation of Microscopic Behaviour for Clay by Using Micro-indenter
}

Kanayama, Motohe i

Agro-environmental Sciences, Faculty of Agriculture, Kyushu University

Ohtsubo, Masami

Agro-environmental Sciences, Faculty of Agriculture, Kyushu University

Higashi, Takahiro

Agro-environmental Sciences, Faculty of Agriculture, Kyushu University

Tanaka, Masanori

Geotechnical and Structural Engineering Department, Port and Airport Research Institute 他

https://doi.org/10.5109/22071

出版情報: 九州大学大学院農学研究院紀要. 57 (1)，pp. 189-194，2012-02. Faculty of Agriculture， Kyushu University

バージョン :

権利関係 : 


\title{
Evaluation of Microscopic Behaviour for Clay by Using Micro-indenter
}

\author{
Motohei KANAYAMA*, Masami OHTSUBO, Takahiro HIGASHI, \\ Masanori TANAKA ${ }^{1}$ and Kenichi SOGA ${ }^{2}$
}

\author{
Laboratory of Environmental Soil Engineering, Division of Bioproduction Environment Sciences, \\ Department of Agro-Environmental Sciences, Faculty of Agriculture, \\ Kyushu University, Fukuoka 812-8581, Japan \\ (Received November 7, 2011 and accepted November 9, 2011)
}

\begin{abstract}
In this study, the shear strength determined by a micro indentation test was examined focusing on the quantitative evaluation of the micro-scale mechanical behaviour of clay. The comparison of the cohesion obtained from the micro indentation test and unconfined compression test for clay samples indicated in the micro indentation test results by cyclic loading underestimate the cohesion while those by monotonic loading estimate the cohesion accurately. It is noted that the loading rate should be selected according to the clay sample because strength is affected by the rate effect. From these results, it was found that the microscopic mechanical behaviour of clay can be evaluated by mean of the micro indentation test.
\end{abstract}

Key words: clay, microscopic behaviour, micro-indentation, rate effect, undrained shear strength

\section{INTRODUCTION}

The macroscopic behaviour of soil ground such as compression and deformation reflects the integrated microscopic behaviour of soil particle movement and pores entrapment under applied pressure. The compression and deformation of soil are the multiple behaviour, since there is the wide variation in the shape and size of soil particle, chemical properties of clay particles and the heterogeneity of pore size distribution. There exist the soil materials which exhibit different mechanical behaviour despite they have the same void ratio. This would be due to difference in the microscopic structure of soil. Hence, it is expected that there is correlation between macroscopic information such as stress-strain relation and microscopic information such as microscopic structure.

Scanning electron microscopy and mercury intrusion porosimetry are both useful methods for the evaluation of microscopic structure of soils. Delage and Lefebvre (1984) presented the results of the scanning electron microscopy and mercury intrusion porosimetry for the freeze-dried samples of natural sensitive clay. They observed clay structure at various stress levels during one-dimensional compression and showed that the collapse of the structure is progressive and first affected is largest interaggregate pores. Griffiths and Joshi (1991) showed from the study on several clayey soils using mercury-intrusion porosimetry that the amount of secondary consolidation varied. They concluded that the secondary consolidation does not occur exclusively as a result of the deformation of micro-pores. Yamaguchi and Ikenaga (1993) showed that the change of pore struc-

${ }^{1}$ Geotechnical and Structural Engineering Department, Port and Airport Research Institute, 3-1-1 Nagase, Yokosuka, 2390826, Japan

${ }^{2}$ Department Engineering, University of Cambridge, Trumpington Street Cambridge, CB2 1PZ, United Kingdom

* Corresponding author (E-mail: motohei@bpes.kyushu-u.ac.jp) ture in the specimens contribute to consolidation, swelling and sand content by using porosimetry. Kang et al. (2002) examined the effect of the drying and preparation procedure of specimen on the micro-structure and pore size distribution of soil for various soil conditions and different testing methods. Based on these results, they clarified the effect of drying process on the consolidation characteristics and pore size distribution for Osaka Pleistocene clay under different loading pressures. Kanayama et al. (2009) conducted conventional consolidation tests on sand-clay mixed soils and examined their pore size distributions and microstructures using mercury-intrusion porosimetry and scanning electron microscope. They found that small intra-aggregate pores are not compressed until all macro pores collapse due to consolidation, and largest interaggregate pores are affected first.

Besides above studies, a number of data on soil structure have been accumulated to explain stress deformation characteristics as macroscopic behaviour. However, there is lack of the quantitative evaluation of correlation between the microscopic and macroscopic behaviour of soil, and hence the data accumulated in the past are not effectively used.

The purpose of this paper is to present the microscopic stress deformation characteristics of clay, and to examine the correlation between the microscopic and macroscopic mechanical properties of clay.

\section{MATERIALS AND METHODS}

\section{Micro-indentation test}

Indentation tests are widely performed to evaluate the properties of solid materials such as strength, wear resistance, and deformation resistance. They are widely used in various industrial areas because the residual life time of a material or structure can be predicted from its hardness value, hardness reduction, or hardness ratio (Ahn and Kwon, 2001; Hill et al., 1989; Kim et al., 2005). 


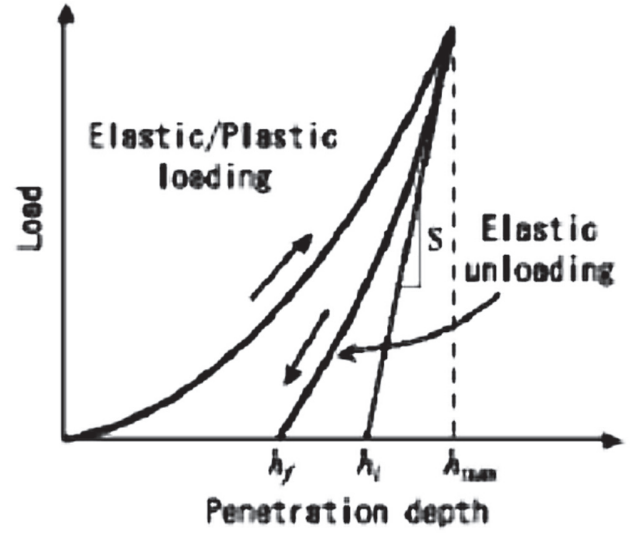

Fig. 1. Schematic depiction of indentation load-depth curves.

Continuous indentation tests have been suggested to be an alternative to hardness tests. In the continuous indentation tests, the indentation load-depth curves are obtained by measuring continuously applied load and penetration depth during testing. Figure 1 shows typical load-depth curves for one loading and unloading. The representative mechanical properties evaluated from the indentation load-depth curves are hardness and elastic modulus. To estimate those values, it is necessary to derive load, $P$, contact area, $A c$, and contact stiffness between indenter and specimen, $S$. The value of $S$ is equivalent to the initial slope of the unloading curve in Figure 1.

\section{Cyclic Penetration Test $(\operatorname{Rod} \phi=3 \mathrm{~mm})$}

In this study, the single column testing system made by Instron \& Co. Ltd was used (Photo 1). The cyclic penetration test means repeated loading and unloading. In this case the adopted indenter rod is a sphere with $3 \mathrm{~mm}$ in diameter. Firstly, to examine the effect of the number of cyclic penetration on the strength of the samples, we adopted 3 loading patterns: 1, 2, 5, 10 and 20 cycles with $1 \mathrm{~mm}$ in maximum penetration depth and $0.1 \mathrm{~mm} / \mathrm{sec}$ in rate, and 40 cycles with $0.8 \mathrm{~mm}$ in maximum penetration depth and $0.1 \mathrm{~mm} / \mathrm{sec}$ in rate. Secondary, to consider the effect of loading rate on the strength of the samples, 6 rates were adopted: $0.0005,0.001,0.01,0.1,1$ and

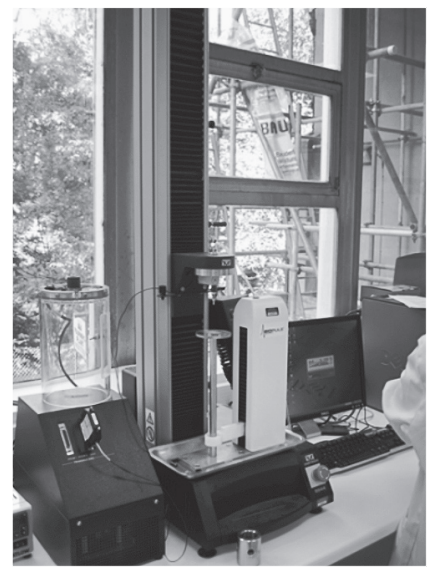

Photo. 1. Single column testing system.
$2 \mathrm{~mm} / \mathrm{sec}$ with $0.5 \mathrm{~mm}$ in maximum penetration depth.

\section{Evaluation of undrained shear strength from micro indentation test results}

Terzaghi revised the plastic solution for the bearing capacity of metal material proposed by Prandtl, and derived the ultimate bearing capacity formula for the general shear failure of the shallow continuous foundation. In this study the results of micro indentation test are transformed to the strength of the samples by using Terzaghi's bearing capacity formula.

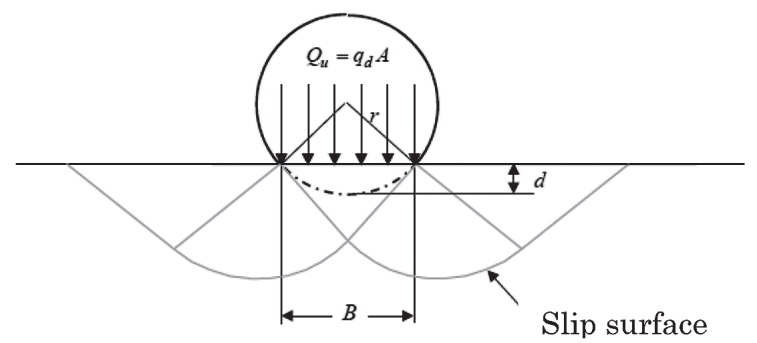

Fig. 2. Bearing capacity of sample.

When the sample is destroyed by the indentation of sphere rod (Figure 2), Terzaghi's formula of equation (1) is applicable for the estimation of the bearing capacity.

$$
Q_{u}=q_{d} A=\frac{\pi B^{2}}{4}\left(1.3 c N_{c}+0.3 \gamma B N_{\gamma}\right)
$$

where $q_{d}$ is the ultimate bearing capacity, $c$ is the cohesion, $\gamma$ is the unit weight of the sample, $B$ is the contact width and $N_{c}, N_{\gamma}$ is the bearing capacity factor.

The parameter $B$ is considered as the contact width when the indent sphere penetrates into the sample surface for only $d$, the diameter that the contact portion is projected on the plane is given as following.

$$
B=2 \sqrt{2 d r-d^{2}} \leq 2 r
$$

The internal friction angle, $\phi$, is assumed to be zero in the case of the clay sample, then $N_{c}$ is 5.14 and $N_{\gamma}$ is 0 , finally the cohesion, $c$ is expressed as equation (3).

$$
c=\frac{Q_{u}}{2 l d(2 r-d)}
$$

The relationship between the unconfined compression strength, $q_{u}$, and the cohesion, $c$, of the sample is generally represented as $q_{u}=2 c$. In this study the $c$ value derived from equation ( 3 ) and the $q_{u} / 2$ value were examined.

\section{Samples}

In the above experiments, the commercially available kaolin clay, and the marine sediment called Ariake clay were used. For the kaolin clay, the remolded sample and the pre-consolidated sample by centrifugal compressor were used. The soil particle density, $\rho_{s}$, the liquid limit, $w_{L}$ and the plastic limit, $w_{p}$, were $2.60 \mathrm{~g} / \mathrm{cm}^{3}, 61 \%$ and $27 \%$, respectively. 
Undisturbed Ariake clay were collected by thin-wall sampler in Saga Prefecture of Kyushu, Japan. The collecting depth is $13.0-13.8 \mathrm{~m}$. The physical properties are as follows. The soil particle density, $\rho_{s}$, was in the range of $2.626-2.638 \mathrm{~g} / \mathrm{cm}^{3}$. The natural water content, $w_{n}$, was in the range of $89.3-91.7 \%$, the liquid limit, $w_{L}$, was in the range $66.6-81.6 \%$, and the plastic limit, $w_{p}$, was in the range $31.3-36.0 \%$. The contents of clay, silt and sand were $46-64 \%, 33-48 \%$ and $3-6 \%$, respectively. The undrained shear strength, $s_{u}$, equivalent to a half of unconfined compression strength was $20.5-32.5 \mathrm{kN} / \mathrm{m}^{2}$.

\section{Unconfined compression test}

Because of simplicity, the unconfined compression test is commonly used to determine the undrained strength of cohesive soil. In this study the unconfined compression test was conducted to compare the results from this test with those from the micro indentation test. The size of specimens was $5 \mathrm{~cm}$ in diameter and $10 \mathrm{~cm}$ in height, and the rate of loading adopted was $0.1 \mathrm{~cm} / \mathrm{min}$ (1\%/min as strain). The amount of compression and load were measured at a designated time, and finally the unconfined compression strength of sample is derived.

Figure 3 shows the result of unconfined compression test for pre-consolidated kaolin clay. The test was conducted in double sets for the samples trimmed from almost the same portion. The unconfined compression strengths, $q_{u}$, were 75.2 and $82.5 \mathrm{kN} / \mathrm{m}^{2}$ at a failure strain of 10.4 and $11.9 \%$, respectively, corresponding to the undrained shear strength of 37.6 and $41.2 \mathrm{kN} / \mathrm{m}^{2}$.

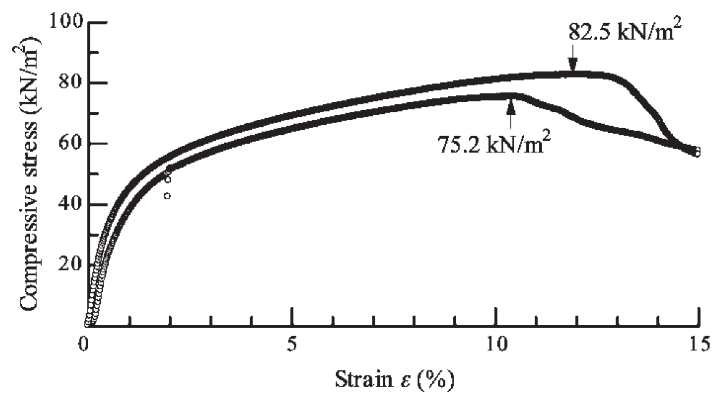

Fig. 3. Result of unconfined compression test for pre-consolidated kaolin clay.

\section{RESULTS AND DISCUSSION}

\section{Results of cyclic penetration test Remolded Kaolin Clay}

The cyclic penetration tests for the remolded kaolin clay with various water contents were conducted and the results for the sample with the water content of $42.2 \%$ are shown in Figure 4. The number of cyclic penetration was set to be 1, 2, 5, 10, 20 and 40 rounds and for each set the depth for one penetration was 1000, 500, 200, 100, 50 and $20 \mu \mathrm{m}$, respectively. The maximum penetration depth for the 40 rounds was $0.8 \mathrm{~mm}$ because of the limitation of the executable code. The load increased almost linearly with increasing the penetration depth and the maximum values of the load were almost the same irrespective of the number of penetration. Thus the number of cyclic penetration does not affect the magnitude of the load. By unloading, the load decreased dramatically and dropped to negative values in any cases. The similar trends were observed for other samples where the load tended to decrease with increasing the water content of the samples.

Figure 5 shows the relationship between the cohesion of the samples calculated by equation (3) and the penetration depth. The cohesion values were in a wide range at the initial stage of penetration, but were almost constant value beyond the penetration of $0.3-0.4 \mathrm{~mm}$. Variation in the cohesion at a given penetration depth tended to be less for the higher water content. This would be ascribed to enhanced homogeneity in the samples with increased water content.

As mentioned above, the cohesion varied to a great extent at the initial stage of penetration for 40 round results. This would be due to transition from the behaviour of individual soil particles to that of a group of soil

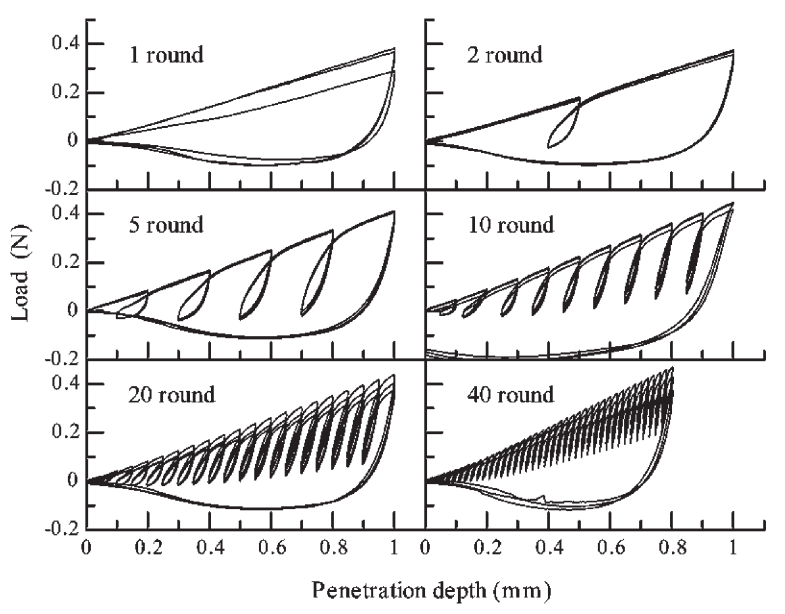

Fig. 4. Load-displacement curves derived from micro indentation test for the remolded kaolin clay ( $w=42.2 \%$ ).

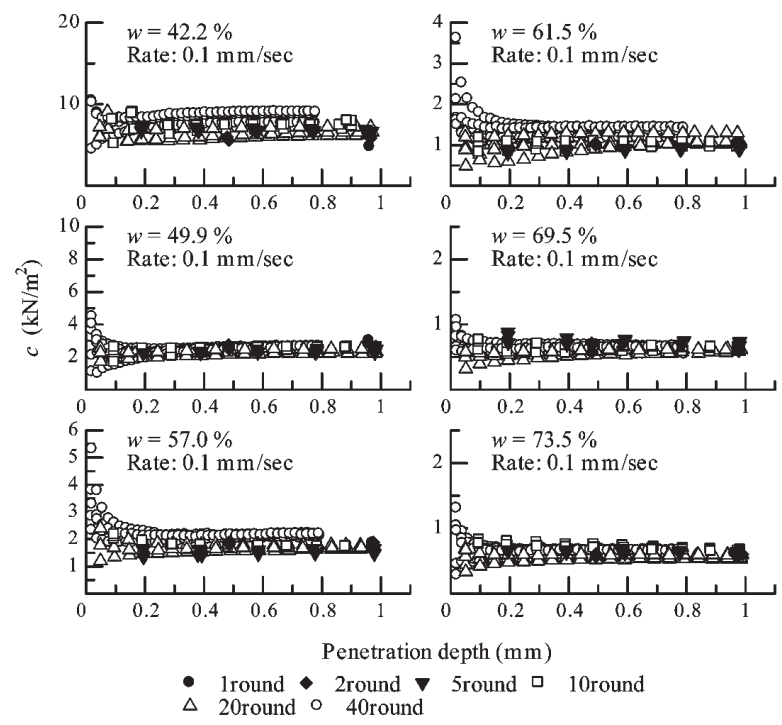

Fig. 5. Relation between the cohesion and penetration depth for the remolded kaolin clay. 


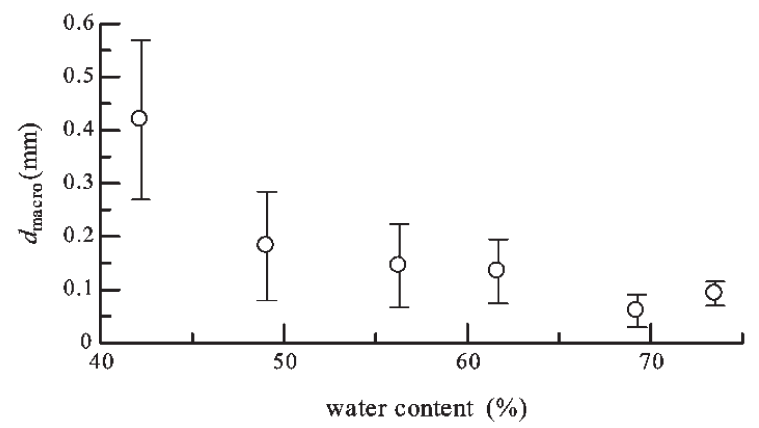

Fig. 6. Relation between $d_{\text {macro }}$ and water content for the remolded kaolin clay.

particles. The penetration depth equivalent to a uniform cohesion was examined focussing on the slope of cohesion and penetration depth. The point that shows a constant value is defined as $d_{\text {macro }}$. Figure 6 shows the relationship between the $d_{\text {macro }}$ and the water content. In this figure the bar denotes the standard deviation. With an increase in the water content of the samples, the standard deviation became small. In the samples with low water content, interparticle distance is small, and the movement of one particle tends to affect adjacent particles. The resultant movement as particle group would need the deeper penetration comparison with the high water content sample. The value of $d_{\text {macro }}$ decreased hyperbolically as the water content increases, because the distance between particles is large and the effect of particle movement on adjacent particles is less.

\section{Pre-consolidated Kaolin Clay}

The pre-consolidated kaolin clay by centrifugation was used to examine the microscopic behaviour of the clay. The water content of the sample was $43.9 \%$, and the number of cyclic penetration was set to be 10, 20 and 40 cycles with the rate of loading of $0.1 \mathrm{~mm} / \mathrm{sec}$. The penetration depth per cycle was 100,50 and $20 \mu \mathrm{m}$ for the sets of the cycles, respectively.

Figure 7 shows the load versus penetration depth curves and the relationship between the cohesion, $c$, derived from equation (3) and penetration depth for the pre-consolidated kaolin clay. In the left figures, the load increased linearly as the penetration depth increases.
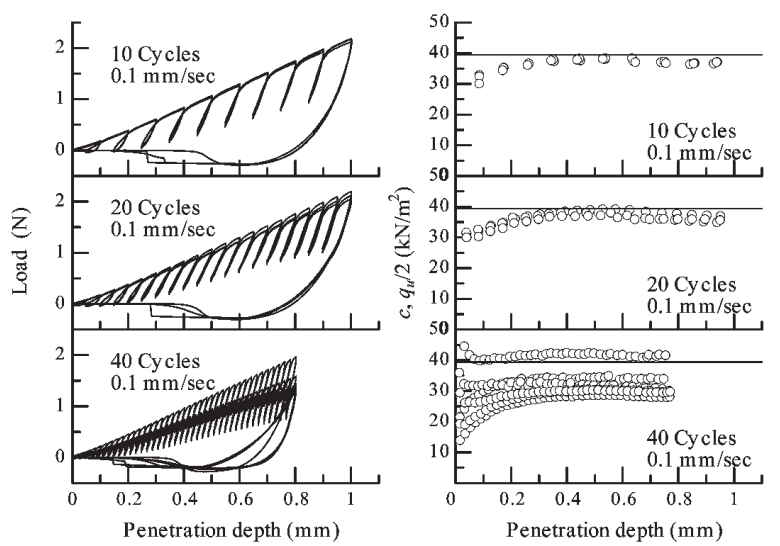

Fig. 7. Microscopic behaviour for pre-consolidated kaolin clay.

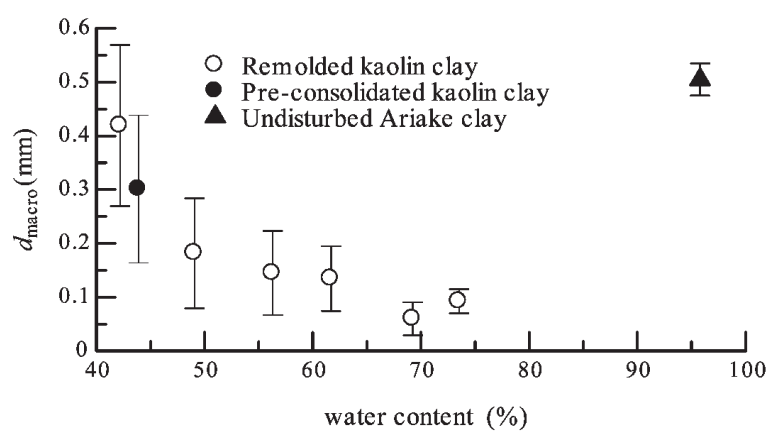

Fig. 8. Relationship between $d_{\text {macro }}$ and water content for the samples.

Variation in the load became large as the cycle of loading increased.

In the right figures, the solid line indicates the undrained shear strength, $q_{u} / 2$. The values of $c$ and $q_{u} / 2$ almost agreed at the penetration depths greater than $0.3 \mathrm{~mm}$ for 10 and 20 cycles. There was large discrepancy between the values of $c$ and $q_{u} / 2$ for 40 cycles. This cause includes various factors as follows: the effect of cyclic number, the effect of inhomogeneity for sample and the installation problem of the sphere rod at the start. As a whole the calculated $c$ value is tend to be slightly underestimation with the $q_{u} / 2$ values.

The indentation depth equivalent to a uniform cohesion, $d_{\text {macro }}$ is examined same as the remolded sample. The result is shown in Figure. 8, and the results of the remolded kaolin clay and the undisturbed Ariake clay are also shown. It is found that the $d_{\text {macro }}$ of the kaolin clay decreases on the same curve line with increasing of the water content regardless of the sample condition, namely remolded or pre-consolidated. Hence, it is identified that the $d_{\text {macro }}$ of the same sample could be expressed as a function of the water content, but a more detailed examination would be needed.

\section{Undisturbed Ariake Clay}

The microscopic behaviour of the undisturbed Ariake clay was examined. The water content of the sample was $95.8 \%$, and the number of cyclic penetration was set to be 10, 20 and 40 rounds with the rate of loading of $0.1 \mathrm{~mm} / \mathrm{sec}$.
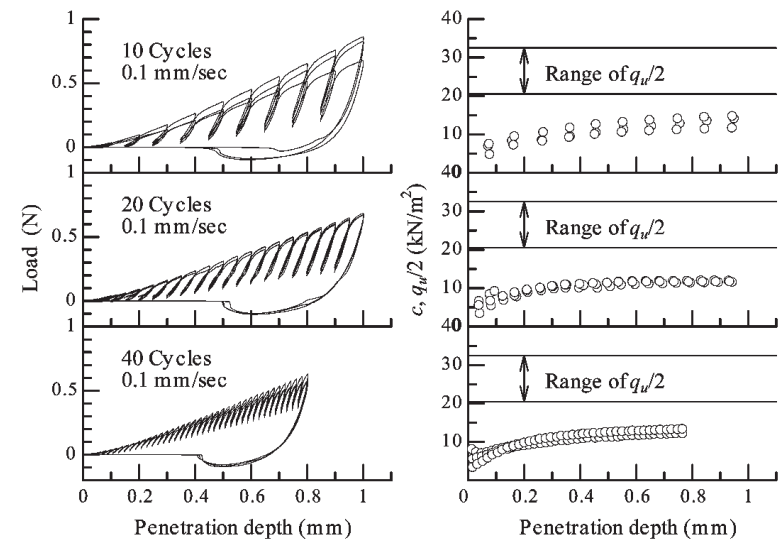

Fig. 9. Microscopic behaviour for undisturbed Ariake clay. 
Figure 9 shows the results of the micro indentation test for the undisturbed Ariake clay. In the load versus penetration curve, the load increased curvedly with increasing penetration depth, which differed in tendency from the kaolin sample where the load increased linearly with increasing the penetration depth (Fig. 7). This may be due to difference in major clay minerals and the presence of diatom and organic matter in the Ariake clay.

The right figure shows the cohesion, $c$, against the penetration depth along with the range of the $q_{u} / 2$ values. The $c$ values increased until the depth reached about $0.5-0.6 \mathrm{~mm}$, and thereafter became almost constant. When the values of $q_{u} / 2$ and $c$ are compared, the $c$ values were smaller than $q_{u} / 2$ values throughout the penetration depth. This indicates that the determination of strength by the micro indenter underestimates the undrained strength of undisturbed clay. This discrepancy might be due to the effect of the adopted indentation rate, that is, the rate effect.

\section{The effect of loading rate on cohesion}

The monotonic indentation test was conducted to examine the effect of loading rate on the cohesion of samples. The test condition was as follows: the maximum indentation depth was $0.5 \mathrm{~mm}$, and the loading rate was $0.0005,0.001,0.01,0.1,1$ and $2 \mathrm{~mm} / \mathrm{sec}$. As the cohesion showed the constant values at the penetration depth about above $0.5 \mathrm{~mm}$, the maximum penetration depth is set to $0.5 \mathrm{~mm}$.

Figure 10 shows the effect of loading rate on the cohesion determined from the monotonic indentation test for pre-consolidated kaolin clay and the undisturbed Ariake clay. Noticing the effect of loading method, namely cyclic and monotonic, the $\mathrm{c}$ value based upon the cyclic loading test is underestimated with that based upon the monotonic loading test. According this result, it is found that the difference in the loading method affects the strength of sample.

For the pre-consolidated kaolin clay the cohesion

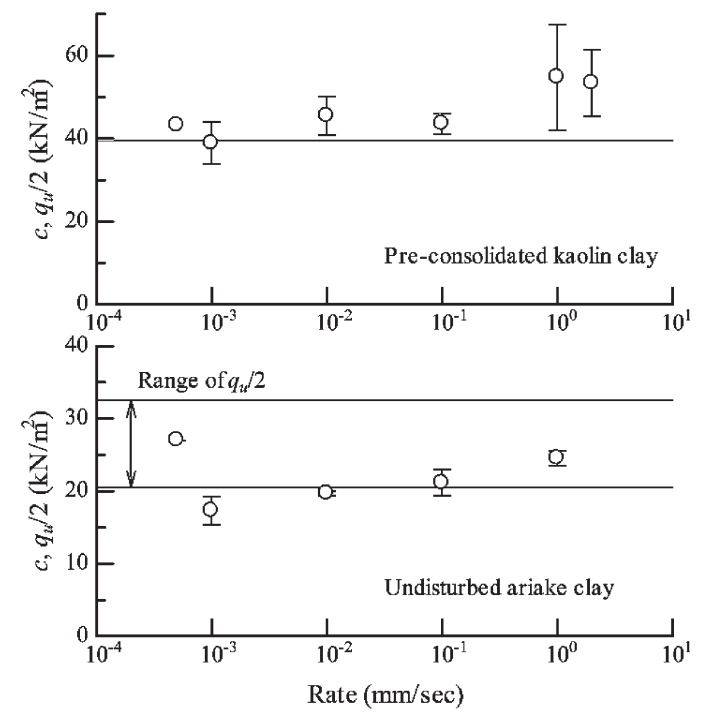

Fig. 10. Rate effect on cohesion derived from micro indentation test. values were almost identical with the $q_{u} / 2$ values at the loading rate less than $0.1 \mathrm{~mm} / \mathrm{sec}$. This indicates that the micro indentation test is valid to evaluate of the kaolinite clay by adopting the loading rate less than $0.1 \mathrm{~mm} / \mathrm{sec}$.

For the undisturbed Ariake clay, the cohesion showed the highest value at $0.0005 \mathrm{~mm} / \mathrm{sec}$, which would be due to the consolidation of the clay. Beyond $0.0005 \mathrm{~mm} / \mathrm{sec}$, the cohesion increased with an increase in the loading rate, which confirms the rate effect of penetration for the Ariake clay. The cohesion values determined at the loading rate above $0.1 \mathrm{~mm} / \mathrm{sec}$ were within the range of the $q_{u} / 2$ values. These results indicate that the cohesion of the undisturbed Ariake clay can be evaluated at the range of 0.1 to $1 \mathrm{~mm} / \mathrm{sec}$ using micro indentation test.

\section{CONCLUSIONS}

In this study, the strength determined from micro indentation test was examined focusing on the quantitative evaluation of micro-scale mechanical behavior of the clay samples, and the microscopic and macroscopic mechanical properties of the clay samples were correlated. The results obtained are in the following.

(1) The micro indentation test is effective to evaluate quantitatively the micro-scale mechanical behavior of clay. But more detailed study is needed on the effect of structural inhomogeneity, clay minerals, diatom and organic matter on micro-scale mechanical behaviour of clay.

(2) The undrained shear strength can be evaluated from the results of microscopic mechanical behaviour obtained by the micro indentation test. Comparing the cohesion, $c$, derived from the micro indentation test with the undrained shear strength, $q_{u} / 2$, derived from unconfined compression test, the resultant $c$ value based upon the cyclic loading test is underestimated and the $c$ value based upon the monotonic loading test shows the almost same value as the $q_{u} / 2$ value. It is noted that the loading rate should be selected in the case of the clay sample that strength affected by the rate effect.

\section{ACKNOWLEDGMENTS}

Financial supports for this study have been provided partially by the Ministry of Education, Culture, Sports, Science and Technology, Japan (Grant in Aid for Scientific Research, No. 23780250).

\section{REFERENCES}

Fred J. Griffiths and Ramesh C. Joshi 1991 Change in pore size distribution owing to secondary consolidation of clays, Canadian Geotechnical Journal, 28: 20-24

H. Yamaguchi and H. Ikenaga 1993 Utilization of mercury intrusion porosimetry apparatus for evaluation of soil structure, Soils and Foundations, 41-4(423): 15-20

J. H. Ahn and D. Kwon 2001 Derivation of plastic stress-strain relationship from ball indenters: Examination of strain definition and pileup effect, Journal of Materials Research, 16: 3170-3178

M. Kanayama, T. Ohira, Y. Ogawa, T. Higashi, M. Ohtsubo and A. Nakano 2009 Variation of Microstructure with Consolidation 
Proceeding for Sand-Clay Mixed Soils, Journal of the Clay Science Society of Japan, 48(1): 1-8

Pierre Delage and Guy Lefebvre 1984 Study of the structure of a sensitive Champlain clay and of its evolution during consolidation, Canadian Geotechnical Journal, 21: 21-35

R. Hill, B. Storakers, A. B. Zdunek 1989 A Theoretical Study of the Brinell Hardness Test, Proceedings of the Royal Society of
London. Series A, Mathematical and Physical Sciences, 423(1865): 301-330

Sung-Hoon Kim, Eun-chae Jeon and Dongil Kwon 2005 Determining Brinell Hardness From Analysis of Indentation Load-Depth Curve Without Optical Measurement, Journal of Engineering Materials and Technology, 127: 154-158 